\title{
CORRECTION
}

\section{Correction to: Prevalence and Risk Factors of Hypertension in the Vietnamese Elderly}

\author{
Nhon Bui Van ${ }^{1}$ Long Vo Hoang ${ }^{2} \cdot$ Tung Bui Van $^{2} \cdot$ Hao Nguyen Si Anh ${ }^{2} \cdot$ Hien Tran Minh ${ }^{2} \cdot \mathrm{Khanh}$ Do Nam ${ }^{2} \cdot$ \\ Tuan Ngo Tri $^{2} \cdot$ Pau Loke Show ${ }^{3} \cdot$ Vu Thi Nga $^{4} \cdot$ Deepak B. Thimiri Govinda Raj ${ }^{5}$. Dinh-Toi Chu ${ }^{6}$
}

Published online: 30 March 2020

(c) Italian Society of Hypertension 2020

\section{Correction to: \\ High Blood Pressure \& Cardiovascular Prevention (2019) 26:239-246 \\ https://doi.org/10.1007/s40292-019-00314-8}

The author affiliations for Deepak B. Thimiri Govinda Raj which previously read:

${ }^{5}$ Institute of Cancer Research, Oslo University Hospital, Oslo, Norway

Should read:

${ }^{5}$ CSIR Synthetic Biology \& Precision Medicine Centre, CSIR Pretoria 0001, South Africa

The original article can be found online at https://doi.org/10.1007/ s40292-019-00314-8.

Vu Thi Nga

nguyenvuphuong.n@gmail.com

$\triangle$ Dinh-Toi Chu

chudinhtoi.hnue@gmail.com

1 Department of Science and Technology, Hanoi Medical University, Hanoi, Vietnam

2 Institute for Preventive Medicine and Public Health, Hanoi Medical University, Hanoi, Vietnam

3 Department of Chemical and Environmental Engineering, Faculty of Engineering, University of Nottingham Malaysia Campus, Jalan Broga, 43500 Semenyih, Selangor Darul Ehsan, Malaysia

4 Institute for Research and Development, Duy Tan University, Danang, Vietnam

5 CSIR Synthetic Biology \& Precision Medicine Centre, CSIR, Pretoria 0001, South Africa

6 Faculty of Biology, Hanoi National University of Education, Hanoi, Vietnam 